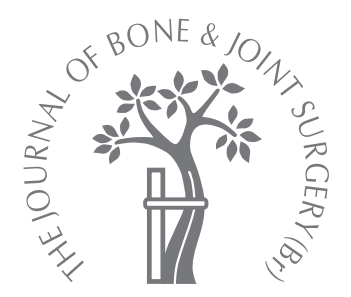

\title{
Giant-cell tumour of the knee
}

\author{
THE CONDITION OF THE CARTILAGE AFTER TREATMENT BY \\ CURETTAGE AND CEMENTING
}

F. Vult von Steyern,

I. Kristiansson,

K. Jonsson,

P. Mannfolk,

D. Heinegård,

A. Rydholm

From Lund

University Hospital,

Lund, Sweden
II F. Vult von Steyern, MD, PhD, Orthopaedic Surgeon

A. Rydholm, MD, PhD,

Professor of Orthopaedic

Oncology

Department of Orthopaedics

I. Kristiansson, MD,

Radiologist

K. Jonsson, MD, PhD,

Radiologist, Professor of Radiology

Department of Diagnostic

Radiology, Centre for Medical

Imaging and Physiology

P. Mannfolk, MSc, PhD

Student

Department of Radiation

Physics

Lund University Hospital,

SE-221 85, Lund, Sweden.

D. Heinegård, MD, PhD,

Professor

Department of Experimental

Medical Sciences

BMC, plan C12, SE-221 84 Lund,

Sweden.

Correspondence should be sent to DrF. Vult von Steyern; e-mail: Fredrik.VultvonSteyern@skane

(C2007 British Editorial Society of Bone and Joint Surgery doi:10.1302/0301-620X.89B3. $18604 \$ 2.00$

$J$ Bone Joint Surg $[\mathrm{Br}]$ 2007:89-B:361-5.

Received 4 September 2006;

Accepted after revision 14

November 2006

We reviewed nine patients at a mean period of 11 years (6 to 16) after curettage and cementing of a giant-cell tumour around the knee to determine if there were any long-term adverse effects on the cartilage. Plain radiography, MRI, delayed gadolinium-enhanced MRI of the cartilage and measurement of the serum level of cartilage oligomeric matrix protein were carried out. The functional outcome was evaluated using the Lysholm knee score.

Each patient was physically active and had returned to their previous occupation. Most participated in recreational sports or exercise.

The mean Lysholm knee score was 92 (83 to 100). Only one patient was found to have cartilage damage adjacent to the cement. This patient had a history of intra-articular fracture and local recurrence, leading to degenerative changes.

Interpretation of the data obtained from delayed gadolinium-enhanced MRI of the cartilage was difficult, with variation in the T1 values which did not correlate with the clinical or radiological findings. We did not find it helpful in the early diagnosis of degeneration of cartilage. We also found no obvious correlation between the serum cartilage oligomeric matrix protein level and the radiological and MR findings, function, time after surgery and the age of the patient.

In summary, we found no evidence that the long-term presence of cement close to the knee joint was associated with the development of degenerative osteoarthritis.

The essential factor in the treatment of a giantcell tumour (GCT) is meticulous curettage of the affected bone. In many centres, the residual defect is filled with polymethylmethacrylate (PMMA) ${ }^{1-4}$ However, some prefer to use bone graft rather than PMMA to avoid the juxtaposition of cement and articular cartilage which could possibly lead to the development of osteoarthritis $(\mathrm{OA}) .^{5,6}$ This is of particular relevance since the most common location of GCT is around the knee. To date, there are no reports on the long-term effects of cement, with respect to degeneration of cartilage, although some studies include occasional cases in which OA has developed. $2,4,7,8$

Delayed Gadolinium-enhanced MRI of cartilage (dGEMRIC) has been used in research to detect early OA. ${ }^{9}$

The technique depends on the established relationship between the load-bearing capacity of articular cartilage and its glycosaminoglycan concentration. The MRI contrast medium (gadolinium diethylene pentaacetic acid) is distributed throughout the articular cartilage in an inverse relationship to the local concentration of glycosaminoglycan. The T1 relaxation rate can then be used to estimate the concentration of the contrast medium from which the local concentration may be calculated.

The presence of cartilage oligomeric matrix protein (COMP) in serum has also been suggested as a possible marker for the diagnoses of early OA. ${ }^{10,11}$ A link has been demonstrated between elevated serum COMP levels and the later appearance of progressive cartilage destruction. ${ }^{12}$ There is, however, an overlap between the levels present in apparently normal individuals and in those with established OA. The levels in a given individual usually remain unchanged with time unless an active cartilage process develops. We have studied the possible development of degeneration of cartilage and $\mathrm{OA}$ in nine patients who had undergone curettage and cementing for GCT around the knee. The patients were examined using plain weight-bearing radiography, MRI, dGEMRIC and measurement of serum COMP levels.

\section{Patients and Methods}

Between 1988 and 1998 we treated 14 patients with GCT around the knee by curettage and 


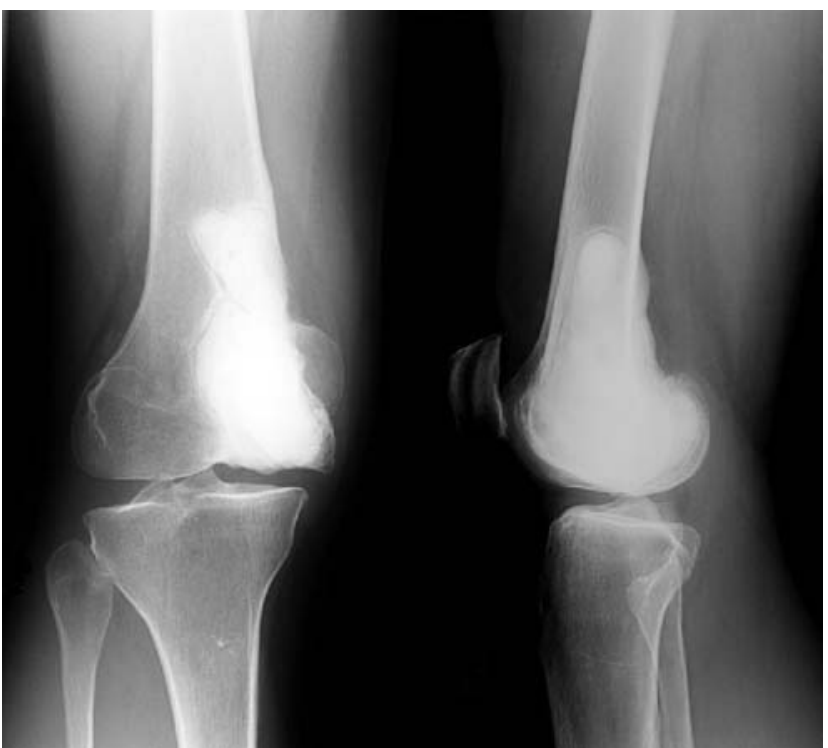

Fig. 1a

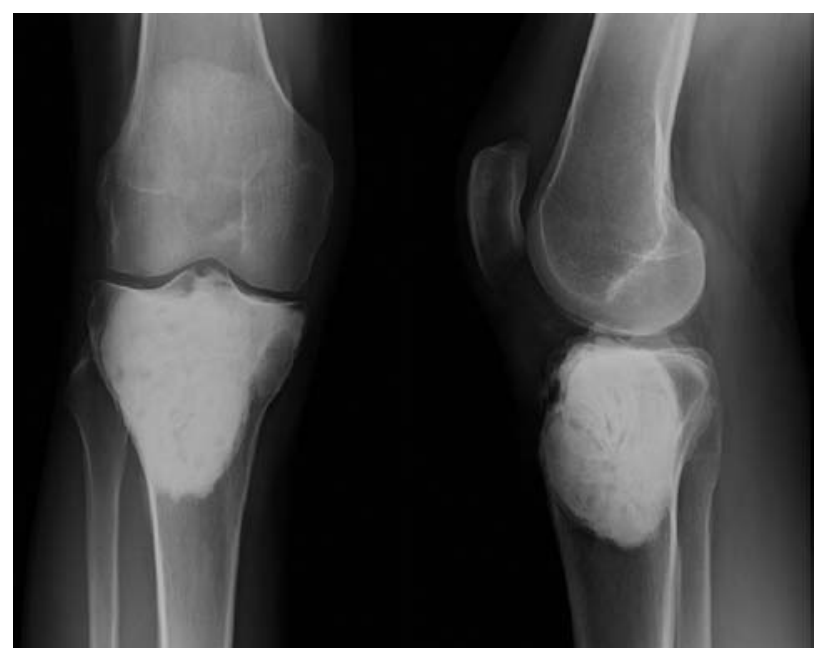

Fig. 1b

Anteroposterior and lateral radiographs of a) case 5 and b) case 6 .

cementing. The lesions were all located close to the articular cartilage (Fig. 1, Table I). The study was approved by the Lund University Ethics Committee. The patients were asked by letter to participate in the study on a voluntary basis. Four failed to reply and one was excluded because of the presence of established OA before the diagnosis of GCT. The nine remaining patients were included in the study at a mean of 11 years (6 to 16) after their first operation. There were three females and six males with a mean age at diagnosis of 29 years (14 to 44 ). The tumour was in the proximal tibia in four and the distal femur in five. The mean maximum diameter of the tumour was $7 \mathrm{~cm}$ (4 to 11) and the mean distance from the cement to the cartilage was $1.0 \mathrm{~mm}$ (0.0 to 3.5) (Fig. 1, Table I). All except one patient had been primarily treated at our hospital and all were free from tumour at the time of follow-up.

After the first operation complications occurred in four patients. The first (case 3) who had a GCT in the distal femur developed pain and a mechanical obstruction to movement in the knee six months later. Small pieces of cement were observed in the joint. No cement had been seen in the joint during operation and he had mobilised normally. Two small pieces of cement ( $6 \mathrm{~mm}$ to $8 \mathrm{~mm}$ ) were removed arthroscopically and a defect was seen in the cartilage in the lateral tibial condyle. Furthermore, 18 months after the first operation he needed further curettage and cementing for local recurrence.

A second patient (case 5) sustained a minimallydisplaced intra-articular fracture of the medial femoral condyle after recurettage and cementing of a local recurrence three months after her primary operation.

The third patient (case 6) had local recurrence 2.5 years after the first operation and was treated by further curettage and cementing. The final patient (case 9) had been treated at another hospital by curettage and bone grafting and was referred 18 months later because of a local recurrence. He, too, was treated by further curettage and cementing.

Each patient was investigated using plain radiography, MRI and measurement of the serum level of COMP. Quantitative measurements were performed in six of the patients using dGEMRIC. In each case the opposite side was used as a reference.

Weight-bearing radiographs, including anteroposterior (AP), lateral and skyline views of the patella, were obtained. Both knees were examined in all nine patients. The minimum distance between the cement margin and the adjacent articular cartilage was measured in the operated knee, as was the minimum joint space in each compartment, in both knees in every patient.

Function was evaluated using the system of Lysholm and Gillquist ${ }^{13}$ (maximum score 100).

MRI of the operated knee was carried out in all the patients using a $3 \mathrm{~T}$ imager (Philips Intera Release 1.2, Philips Medical Systems, Best, The Netherlands) with a SENSE-flex medium coil (Philips Medical Systems). In six patients dGEMRIC of both knees was undertaken.

The T1 relaxation rate measurements were performed before and after injection of contrast using an inversion recovery pulse sequence with different inversion times $(50,100,200,400,800$ milliseconds (ms); and 1600 sagittal slices with thickness $5 \mathrm{~mm}, \mathrm{TR}=2000 \mathrm{~ms}$, TE $=15$ $\mathrm{ms}$, turbo factor 11, field of view $(\mathrm{FoV})=120 \mathrm{~mm} \times 120$ $\mathrm{mm}$, matrix size $=256 \times 204)$.

In order to allow the contrast medium to penetrate, the patients were instructed to walk for 15 minutes after a triple dose injection of contrast $(0.3 \mathrm{mmol} / \mathrm{kg}$ bodyweight) before carrying out the post-contrast measurement 90 minutes after the injection. 
Table I. Details of the nine patients

\begin{tabular}{|c|c|c|c|c|c|c|c|c|c|c|}
\hline Case & $\begin{array}{l}\text { Age } \\
\text { (yrs) }\end{array}$ & Gender & Location & $\begin{array}{l}\text { Size } \\
\text { (cm) }\end{array}$ & Fracture & Local recurrence & $\begin{array}{l}\text { Follow-up } \\
\text { (yrs) }\end{array}$ & Lysholm score & $\begin{array}{l}\text { COMP* serum }^{*} \\
\text { levels (U/L) }\end{array}$ & $\begin{array}{l}\text { Cement-cartilage } \\
\text { distance }(\mathrm{mm})\end{array}$ \\
\hline 1 & 18 & $\mathrm{~F}$ & Tibia & 5 & No & No & 11 & 83 & 8.3 & 0.5 \\
\hline 2 & 14 & $\mathrm{~F}$ & Femur & 11 & Yes & No & 15 & 95 & 11.4 & 3.5 \\
\hline 3 & 20 & $M$ & Femur & 9 & No & Yes & 12 & 90 & 6.8 & 1.5 \\
\hline 4 & 28 & $M$ & Femur & 7 & No & No & 9 & 94 & 10.4 & 1.0 \\
\hline 5 & 35 & $\mathrm{~F}$ & Femur & 7 & $\mathrm{No}^{\dagger}$ & Yes & 6 & 89 & 15.1 & 0.0 \\
\hline 6 & 34 & $M$ & Tibia & 5 & No & Yes & 8 & 100 & 14.8 & 0.0 \\
\hline 7 & 33 & M & Tibia & 9 & No & No & 10 & 98 & 7.6 & 1.0 \\
\hline 8 & 34 & M & Femur & 4 & No & No & 16 & 88 & 14.1 & 0.0 \\
\hline 9 & 44 & $M$ & Tibia & 6 & No & Yes $^{\ddagger}$ & 10 & 92 & 13.3 & 1.5 \\
\hline
\end{tabular}

* COMP, cartilage oligomeric matrix protein

$\dagger$ fracture after surgery for local recurrence

$\neq$ local recurrence at referral

Table II. T1 relaxation time in the dGEMRIC ${ }^{*}$ experiments

\begin{tabular}{|c|c|c|c|c|}
\hline \multirow[b]{2}{*}{ Case } & \multicolumn{2}{|c|}{ Operated kneet } & \multicolumn{2}{|c|}{ Contralateral knee } \\
\hline & T1 pre (ms) & T1 post (ms) & T1 pre (ms) & T1 post (ms) \\
\hline 3 & 764 & 503 & 1031 & 475 \\
\hline 4 & 1232 & 220 & 1235 & 557 \\
\hline 5 & 2650 & 168 & 1305 & 477 \\
\hline 6 & 1233 & 811 & 1211 & 513 \\
\hline 7 & 1211 & 406 & 988 & 273 \\
\hline 9 & 2783 & 749 & 503 & 92 \\
\hline
\end{tabular}

The dGEMRIC measurements were made in a region of interest in each knee which was determined by identifying the area with the shortest distance between the cement and the articular cartilage. In the unaffected knee this was sited in the corresponding area of cartilage and used as a reference. The same radiologist (IK) carried out all the studies.

The serum COMP level was measured using a commercially-available sandwich-based ELISA (Anamar Medical Co., Gothenburg, Sweden).

\section{Results}

Post-operative function was generally good. The mean Lysholm knee score was 92 (83 to 100) (Table I). All the patients were physically active, had returned to their previous occupations and were able to perform recreational sport or exercise. One patient had a Lysholm score of 83 but no radiological signs of OA, although MRI revealed minor degenerative change in the medial meniscus.

Morphology by conventional radiography and MRI. One patient with a post-operative fracture had an irregular contour of the medial femoral condyle, and localised narrowing of the joint space on conventional radiography (Fig. 1a). On MRI there was an area measuring $15 \mathrm{~mm}$ in diameter in which there was no visible cortical bone covering the cement although the cartilage was still present. No other patient showed signs of degeneration of cartilage or of OA on conventional weight-bearing radiographs.
In two patients, MRI showed subtle signs of cartilage disorder. In one there was an irregularity of the femoral cartilage adjacent to the cement. As described previously, the cement had penetrated the joint and a minor cartilage defect was visible in the tibial condyle. Ten years had passed since the cartilage lesion had been verified arthroscopically and there seemed to have been no further deterioration in the cartilage. In the other patient MRI showed cement protruding into the articular cartilage, but no other signs of damage.

The pre-contrast $\mathrm{T} 1$ relaxation rate values varied widely with a mean of $1645 \mathrm{~ms}$ (764 to 2783) in the operated knees and of $1045 \mathrm{~ms}$ (503 to 1305) in the non-operated knees. The post-contrast values varied with a mean of $476 \mathrm{~ms}$ (168 to 811) in the operated knees and $398 \mathrm{~ms}(92$ to 557 ) in the other side. The low post-contrast T1 values would indicate a loss of glycosaminoglycans. In one patient (case 5) the pre-contrast value was high and the post-contrast value low on the operated side when compared with most of the other patients and with the opposite knee, indicating degeneration of the cartilage (Table II). This agreed with the findings on conventional radiography, MRI and with the serum COMP level, all of which suggested the onset of OA, which subsequently developed.

In the remaining patients we could not relate the results from the dGEMRIC to any obvious signs of early OA, although there was some correlation between the pre-contrast T1 relaxation rate value and the serum COMP level. However, no such correlation was observed for the post-contrast value which would give a more accurate indication of cartilage degeneration (Tables I and II).

Some of the serum COMP levels appeared to be high (Table I). However, except in one patient (case 5) in which a reduced joint space on conventional radiography, MRI, dGEMRIC and COMP all indicated cartilage degeneration, we could not see any clear correlation between COMP values and other factors. We found no correlation between these relatively high values and any other factors. Moreover, in the patient in whom cement had protruded into the cartilage without signs of cartilage degeneration, there was a relatively high level of the serum COMP (mean 11.3 U/L (6.8 to 15.1)). 
We found no obvious correlation between the serum COMP level and age, time from diagnosis and surgery, radiological findings, dGEMRIC and functional outcome.

\section{Discussion}

The possible adverse effect of acrylic cement on articular cartilage after curettage and cementing of a GCT has not been fully assessed. Earlier published series of GCTs treated by curettage and cementing record only a few cases of degenerative change which were not necessarily related either to the surgery or to the cement. ${ }^{2,8}$ In a recent report Lackman et $\mathrm{al}^{4}$ described 63 patients treated for GCT by curettage and cementing, of whom 46 had lesions either in the distal femur or in the proximal tibia. After a mean follow-up of nine years one patient had developed OA. Moreover, patients with radiological signs of OA pre-operatively did not deteriorate substantially after their surgery. However, no weight-bearing radiographs had routinely been obtained and evaluation of OA was therefore somewhat basic.

In our study, OA was observed on weight-bearing radiographs in one patient, in whom an intra-articular fracture had occurred after surgery for local recurrence.

The use of dGEMRIC has been suggested as a possible method of detecting early OA. ${ }^{9}$ However, it is not routinely employed in clinical practice and more studies are needed to establish its efficacy. Our results were not easy to interpret and the data obtained varied to a large extent without any obvious relation to other factors. In a study of dGEMRIC in early OA of the knee Tiderius et al, ${ }^{9}$ chose a region of interest in the midline of the condyles of the knee where the cartilage is best outlined and supposedly thickest. Giant-cell tumours tend to be located eccentrically in the distal femur or proximal tibia. Consequently, in our study we measured the $\mathrm{T} 1$ relaxation rate at the point where the distance between the bone cement and the articular cartilage was least. In most cases this was at the edge of the condyles where the cartilage was thin and thus hampered the selection of a region of interest which included only cartilage; this may explain the variation in the values. Apart from the results in one patient, in whom dGEMRIC may have indicated degeneration of cartilage, this method did not confirm or exclude the possible presence of early OA.

The level of the serum COMP has been used as a marker for evaluating degeneration of cartilage and OA. ${ }^{10,11}$ Petersson et $\mathrm{al}^{10}$ noted that the baseline levels between groups of individuals without radiological signs of OA did not differ. However, after three years, patients who had developed signs of OA also had a raised serum COMP level. Diurnal variation in the COMP level occurs. ${ }^{14,15}$ However, when sampling was performed at four-hour intervals throughout a day and night, it was noted that COMP levels were constant during the day. ${ }^{15}$ Therefore, samples can be reproducibly analysed during the daytime when the patients are mobile, as in the present study.
In our study we had only baseline values of the serum COMP at the outset. There was no longitudinal follow-up and the level of activity before sampling was not standardised. We do not, however, believe that these factors significantly affected our results. A level of serum COMP of around $15 \mathrm{U} / \mathrm{L}$ or more is above the $95 \%$ percentile in a population of blood donors and in this relatively young group, the levels in four patients are towards the high end. ${ }^{16}$ One patient apparently had an ongoing process in the cartilage which led to the morphological changes observed. It is possible that another who showed cement protruding into the joint cartilage, had altered cartilage turnover. Further follow-up will be of interest in two patients as to whether an altered cartilage metabolism or elevated levels indicate the early phase of degenerative change. The long interval between surgery and follow-up without clinical signs of $\mathrm{OA}$ suggests that if there is a pathological process leading to OA it is well compensated for by continuous remodelling. Apart from the results in one patient we were unable to distinguish a pattern in which the levels of the serum COMP could be correlated with any other measurement such as age, time from diagnosis or surgery, morphological findings and function.

The role of bone in the development of OA is not clear. It has always been assumed that the subchondral sclerosis seen in OA is the result of increased bone density and stiffness. This must now be questioned given that there appears to be a reduction in bone density and in other aspects of mineralisation of tissues, e.g. cartilage and trabecular bone. $^{17}$

A finite-element study of the femoral head and neck, ${ }^{18}$ suggested that stiffening of the subchondral plate plays an important mechanical role in the degeneration of cartilage. Frassica et $\mathrm{al}^{19}$ have shown that in dogs, replacing subchondral bone with cement does not increase its stiffness. The cement, however, does serve as a stable subchondral platform which may be an important factor for avoiding cartilage degeneration. In our series even the patients with little or no space between the cement and cartilage did not appear prone to develop OA, despite the altered mechanical and physiological environment. Another factor is the effect of altering the blood supply but this is likely to be of less significance since the cartilage is principally nourished by the synovium and, as long as this remains intact, cartilage nutrition will be maintained.

This is a small series with a long follow-up. We have found no evidence to support the view that the treatment of GCT by curettage and cementing induces degeneration of cartilage leading to OA. In our opinion it remains a safe method of treatment even if the tumour involves the subchondral bone of the knee.

This study was supported financially by the Capio Research Foundation, Swe den, the Swedish Children's Cancer Foundation and Region Skåne, Lund University Hospital, Sweden.

No benefits in any form have been received or will be received from a commercial party related directly or indirectly to the subject of this article. 


\section{References}

1. O'Donnel RJ, Springfield DS, Motwani HK, et al. Recurrence of giant-cell tumors of the long bones after curettage and packing with cement. J Bone Joint Surg [Am] 1994;76-A:1827-33.

2. Dreinhöfer KE, Rydholm A, Bauer HC, Kreicbergs A. Giant-cell tumours with fracture at diagnosis: curettage and acrylic cementing in ten cases. J Bone Joint Surg [Br] 1995;77-B:189-93.

3. Saiz P, Virkus W, Piasecki P, et al. Results of giant cell tumor of bone treated with intralesional excision. Clin Orthop 2004;424:221-6.

4. Lackman RD, Hosalkar HS, Ogilvie CM, Torbert JT, Fox EJ. Intralesional curettage for grades II and III giant cell tumours of bone. Clin Orthop 2005;438:123-7.

5. Blackley HR, Wunder JS, Davis AM, et al. Treatment of giant-cell tumours of long bones with curettage and bone-grafting. J Bone Joint Surg [Am] 1999;81A:811-20.

6. Zhen W, Yaotian H, Songjian L, Ge L, Quingliang W. Giant-cell tumor of bone: the long-term results of treatment by curettage and bone graft. $J$ Bone Joint Surg [Br] 2004;86-B:212-16.

7. Bini SA, Gill K, Johnston J0. Giant cell tumor of bone: curettage and cement reconstruction. Clin Orthop 1995;321:245-50.

8. Wada T, Kaya M, Nagoya S, et al. Complications assocated with bone cementing for the treatment of giant cell tumors of bone. J Orthop Sci 2002;7:194-8.

9. Tiderius CJ, OIsson LE, Leander P, Ekberg O, Dahlberg L. Delayed gadolinium-enhanced MRI of cartilage (dGEMRIC) in early knee osteoarthritis. Magn Reson Med 2003;49:488-92.
10. Petersson IF, Boegard T, Svensson B, Heinegard D, Saxne T. Changes in cartilage and bone metabolism identified by serum markers in early osteoarthritis of the knee joint. Br J Rheumato/ 1998;37:46-50.

11. Vilium V, Olejarova M, Machacek S, et al. Serum levels of cartilage oligomeric matrix protein (COMP) correlate with radiographic progression of knee osteoarthritis. Osteoarthritis Cartilage 2002;10:707-13.

12. Sharif M, Saxne T, Shepstone L, et al. Relationship between serum cartilage oligomeric matrix protein levels and disease progression in osteoarthritis of the knee joint. $\mathrm{Br}$ $J$ Rheumatol 1995;34:306-10.

13. Lysholm J, Gillquist J. Evaluation of knee ligament surgery results with special emphasis on use of a scoring scale. Am J Sports Med 1982;10:150-4.

14. Kong SY, Stabler TV, Criscione LG, et al. Diurnal variation of serum and urine biomarkers in patients with radiographic knee osteoarthritis. Arthritis Rheum 2006:54:2496504

5. Andersson ML Petersson IF Karlsson KE et al. Diurnal variation in serum levels of cartilage oligomeric matrix protein in patients with knee osteoarthritis or rheumatoid arthritis. Ann Rheum Dis 2006;65:1490-4.

16. No authors listed. Anamar Medical Co., Sweden. www.anamar.com (date last accessed 14 February 2007).

17. Burr DB. The importance of subchondral bone in the progression of osteoarthritis. $J$ Rheumatol Supp/ 2004;70:77-80.

18. Wei HW, Sun SS, Jao JH, Yeh CR, Cheng CK. The influence of mechanical properties of subchondral plate, femoral head and neck on dynamic stress distribution of the articular cartilage. Med Eng Phys 2005;27:295-304.

19. Frassica FJ, Gorski JP, Pritchard DJ, Sim FH, Chao EY. A comparative analysis of subchondral replacement with polymethylmethacrylate or autogenous bone grafts in dogs. Clin Orthop 1993;293:378-90. 